\title{
CLASSICAL AND NEOCLASSICAL TRANSPORT IN TOKAMAKS
}

\author{
P. Helander \\ Max Planck Institute for Plasma Physics \\ Wendelsteinstraße 1, 17491 Greifswald, Germany
}

\section{ABSTRACT}

These lecture notes provide a short overview of classical and neoclassical transport in tokamaks. The classical theory is widely applicable in laboratory and space plasma physics if the mean free path is shorter than the macroscopic scale length. The neoclassical theory predicts important phenomena in tokamaks such as the bootstrap current, electric conductivity, transport in the scrape-off layer, and cross-field transport in regions where the turbulence is suppressed.

\section{INTRODUCTION}

After Boltzmann had formulated his famous kinetic equation for dilute gases in the 1870's, an outstanding problem in theoretical physics was to calculate transport coefficients (heat conductivity and viscosity) from it. This problem remained open for over four decades until Chapman [1] and Enskog [2] independently found the correct asymptotic expansion. The extension of their technique to ionised gases is referred to as classical or neoclassical theory, depending on whether the geometry of the magnetic field plays a role. In either case, it is a rigorous theory, meaning that it provides an asymptotic solution of the kinetic equation

$$
\frac{\partial f_{a}}{\partial t}+\mathbf{v} \cdot \nabla f_{a}+\frac{e_{a}}{m_{a}}(\mathbf{E}+\mathbf{v} \times \mathbf{B}) \cdot \frac{\partial f_{a}}{\partial \mathbf{v}}=C_{a}\left(f_{a}\right)
$$

for each species $a$, under the assumption that the gyroradius parameter $\delta=\rho_{a} / L$ is small, where $\rho_{a}$ is the gyroradius and $L$ the macroscopic scale length. In classical transport theory, it is assumed that the collisional mean free path $\lambda$ is short, $\lambda / L \ll 1$, whilst in neoclassical theory $\lambda$ is arbitrary. The extension to long mean free path is possible because a transport ordering is adopted, meaning that the time derivative is considered small,

$$
\partial / \partial t \sim \delta^{2} v_{T a} / L
$$

where $v_{T a}=\left(2 T_{a} / m_{a}\right)^{1 / 2}$ is the thermal speed. This ordering removes plasma waves from the equations.
There is one final assumption concerning the magnitude of the electric field. This can either be ordered large,

$$
E / B \sim v_{T a}
$$

or small,

$$
E / B \sim \delta v_{T a},
$$

and the resulting theory is somewhat different in the two cases. Most commonly, the first ordering is adopted in the classical theory and the second one in the neoclassical theory.

\section{FLUID EQUATIONS}

As is well known, the moments of (1) express the conservation of density, momentum and energy,

$$
\begin{gathered}
\frac{\partial n_{a}}{\partial t}+\nabla \cdot\left(n_{a} \mathbf{V}_{a}\right)=0 \\
\frac{\partial\left(m_{a} n_{a} \mathbf{V}_{a}\right)}{\partial t}+\nabla \cdot \mathbf{\Pi}_{a}=n_{a} e_{a}\left(\mathbf{E}_{a}+\mathbf{V}_{a} \times \mathbf{B}\right)+\mathbf{F}_{a} \\
\frac{\partial}{\partial t}\left(\frac{3 n_{a} T_{a}}{2}+\frac{m_{a} n_{a} V_{a}^{2}}{2}\right)+\nabla \cdot \mathbf{Q}_{a} \\
=e_{a} n_{a} \mathbf{E} \cdot \mathbf{V}_{a}+\int \frac{m_{a} v^{2}}{2} C_{a}\left(f_{a}\right) d^{3} v
\end{gathered}
$$

and it is the task of the kinetic theory to calculate the friction force

$$
\mathbf{F}_{a}=\int m_{a} \mathbf{v} C_{a}\left(f_{a}\right) d^{3} v
$$

the pressure tensor

$$
\boldsymbol{\Pi}_{a}=\int m_{a} \mathbf{v} \mathbf{v} f_{a} d^{3} v
$$

the energy flux

$$
\mathbf{Q}_{a}=\int \frac{1}{2} m_{a} v^{2} \mathbf{v} f_{a} d^{3} v
$$

and the collisional energy exchange on the right-hand side of (4). The viscosity tensor is defined as

$$
\boldsymbol{\pi}_{a}=\boldsymbol{\Pi}_{a}-p_{a} \mathbf{I}-m_{a} n_{a} \mathbf{V}_{a} \mathbf{V}_{a}
$$


where $\mathbf{I}$ is the unit tensor, and the heat flux is

$$
\mathbf{q}_{a}=\mathbf{Q}_{a}-\frac{5 p_{a} \mathbf{V}_{a}}{2}-\boldsymbol{\pi}_{a} \cdot \mathbf{V}_{a}-\frac{m_{a} n_{a} V_{a}^{2} \mathbf{V}_{a}}{2}
$$

The collision operator

$$
C_{a}=\sum_{a} C_{a b}\left(f_{a}, f_{b}\right)
$$

is a sum of contributions from collisions with each particle species " $b$ ", including $b=a$. Its detailed form will be of little concern to us, but it is important to know that it conserves particles,

$$
\int C_{a b}\left(f_{a}, f_{b}\right) d^{3} v=0
$$

which has been used in the derivation of the continuity equation (2), as well as momentum and energy,

$$
\begin{aligned}
& \int m_{a} \mathbf{v} C_{a b}\left(f_{a}, f_{b}\right) d^{3} v=-\int m_{b} \mathbf{v} C_{b a}\left(f_{b}, f_{a}\right) d^{3} v \\
& \int \frac{m_{a} v^{2}}{2} C_{a b}\left(f_{a}, f_{b}\right) d^{3} v=-\int \frac{m_{b} v^{2}}{2} C_{b a}\left(f_{b}, f_{a}\right) d^{3} v
\end{aligned}
$$

It will also be useful to know that the operator contains pitch-angle scattering,

$$
\text { a part of } C_{a b}\left(f_{a}\right)=\nu_{a b} \mathcal{L}\left(f_{a}\right)
$$

where the scattering frequency $\nu_{a b}$ is of the order of the inverse collision time, and the scattering operator is defined by

$$
\mathcal{L}=\frac{1}{2} \frac{\partial}{\partial \xi}\left(1-\xi^{2}\right) \frac{\partial}{\partial \xi}
$$

with $\xi=v_{\|} / v$.

\section{CLASSICAL TRANSPORT THEORY}

This theory was developed independently by several authors, but the most well-known and pedagogic formulation is due to Braginskii [3]. It considers a plasma with a single ion species $i$, but can straightforwardly be extended to multiple ion species, and assumes the large-flow ordering, $E / B \sim v_{T i}$. It is remarkable that the complete theory for the small-flow case was only worked out only half a century after Braginskii's original work [4].

\section{III.A. Chapman-Enskog expansion procedure}

Since the flow is considered large, the first step is to transform the kinetic equation (1) to a coordinate frame moving with the flow velocity $\mathbf{V}_{a}(\mathbf{r}, t)$

$$
\frac{d f_{a}}{d t}+\mathbf{v}_{a}^{\prime} \cdot \nabla f_{a}+\left[\frac{e_{a}}{m_{a}}\left(\mathbf{E}^{\prime}+\mathbf{v}_{a}^{\prime} \times \mathbf{B}\right)-\frac{d \mathbf{V}_{a}}{d t}\right] \cdot \frac{\partial f_{a}}{\partial \mathbf{v}_{a}^{\prime}}
$$

$$
-v_{a j}^{\prime} \frac{\partial V_{a k}}{\partial r_{j}} \frac{\partial f_{a}}{\partial v_{a k}^{\prime}}=C_{a}\left(f_{a}\right)
$$

where $\mathbf{v}_{a}^{\prime}=\mathbf{v}-\mathbf{V}_{a}, \mathbf{E}^{\prime}=\mathbf{E}+\mathbf{V}_{a} \times \mathbf{B}$ is the electric field measured in the moving frame, and

$$
\frac{d}{d t}=\frac{\partial}{\partial t}+\mathbf{V}_{a} \cdot \nabla
$$

is the convective derivative. The largest terms are those involving the collision operator and the magnetic field, and the equation can be solved perturbatively by expanding the distribution function accordingly, $f_{a}=f_{a 0}+f_{a 1}+\ldots$. In lowest order, the large terms force the electron $\left(e_{e}=-e\right)$ distribution function to be a Maxwellian at rest in the moving frame,

$$
f_{e 0}=n_{e}\left(\frac{m_{e}}{2 \pi T_{e}}\right)^{3 / 2} e^{-x^{2}}
$$

where $x^{2}=m_{e} v_{e}^{\prime 2} / 2 T_{e}$. In the next order, we obtain an equation for the correction $f_{e 1}$ to the Maxwellian

$$
\begin{gathered}
C_{e e}\left(f_{e 1}\right)+C_{e i}^{0}\left(f_{e 1}\right)+\frac{e}{m_{e}} \mathbf{v} \times \mathbf{B} \cdot \frac{\partial f_{e 1}}{\partial \mathbf{v}} \\
=\left[\frac{d \ln n_{e}}{d t}+\left(x^{2}-\frac{3}{2}\right) \frac{d \ln T_{e}}{d t}+\mathbf{v} \cdot \nabla \ln n_{e}\right. \\
+\left(x^{2}-\frac{3}{2}\right) \mathbf{v} \cdot \nabla \ln T_{e}+\frac{m_{e} \mathbf{v}}{T_{e}} \cdot\left(\frac{e}{m_{e}} \mathbf{E}^{\prime}+\frac{d \mathbf{V}_{e}}{d t}\right) \\
\left.+\frac{m_{e} v_{j} v_{k}}{T_{e}} \frac{\partial V_{e k}}{\partial r_{j}}+\nu_{e i} \frac{m_{e} \mathbf{v} \cdot \mathbf{u}}{T_{e}}\right] f_{e 0},
\end{gathered}
$$

where we have now written $\mathbf{v}$ instead of $\mathbf{v}_{e}^{\prime}$ and where $\mathbf{u}=\mathbf{V}_{e}-\mathbf{V}_{i}$ is the mean velocity of the electrons relative to the ions. $C_{e i}^{0}\left(f_{e 1}\right)=\nu_{e i} \mathcal{L}\left(f_{e 1}\right)$ denotes the scattering part of the electron-ion collision operator.

A crucial insight by Chapman and Enskog was that the time derivatives on the right-hand side can be eliminated by taking moments of the equation. Indeed, integrating Eq (9) over velocity space gives the continuity equation (2), which implies that $d \ln n_{e} / d t$ can be replaced by $-\nabla \cdot \mathbf{V}_{e}$. The $m_{e} \mathbf{v}$-moment gives the momentum equation (3), but without the viscosity term, and can be used to eliminate $d \mathbf{V}_{e} / d t$ and $\mathbf{E}^{\prime}$ by

$$
\frac{d \mathbf{V}_{e}}{d t}+\frac{e \mathbf{E}^{\prime}}{m_{e}}=\frac{\mathbf{F}_{e}-\nabla\left(n_{e} T_{e}\right)}{m_{e} n_{e}} .
$$

The energy moment, finally, gives the energy equation (4), but without heat conduction and energy exchange,

$$
\frac{3}{2} \frac{d \ln T_{e}}{d t}+\nabla \cdot \mathbf{V}_{e}=0
$$

and this can be used to eliminate $d T_{e} / d t$ in Eq (9). The reason why certain terms in the full fluid equations (2)(4) do not appear in the moments of Eq (9) is that 
they are small in the ordering assumed. Eliminating time derivatives from $\mathrm{Eq}(9)$ in this manner gives the following kinetic equation for $f_{e 1}$ in the electron rest frame,

$$
\begin{gathered}
C_{e e}\left(f_{e 1}\right)+C_{e i}^{0}\left(f_{e 1}\right)+\frac{e}{m_{e}} \mathbf{v} \times \mathbf{B} \cdot \frac{\partial f_{e 1}}{\partial \mathbf{v}} \\
=\left[\left(x^{2}-\frac{5}{2}\right) \mathbf{v} \cdot \nabla \ln T_{e}+\mathbf{v} \cdot\left(\frac{\mathbf{F}_{e}}{p_{e}}+\frac{m_{e} \nu_{e i} \mathbf{u}}{T_{e}}\right)\right. \\
\left.+\frac{m_{e}}{2 T_{e}}\left(v_{j} v_{k}-\frac{v^{2}}{3} \delta_{j k}\right) W_{j k}^{e}\right] f_{e 0},
\end{gathered}
$$

where

$$
W_{j k}^{a} \equiv \frac{\partial V_{a j}}{\partial r_{k}}+\frac{\partial V_{a k}}{\partial r_{j}}-\frac{2}{3}\left(\nabla \cdot \mathbf{V}_{a}\right) \delta_{j k} .
$$

is the so-called rate-of-strain tensor. Note that there are three driving terms on the right: the temperature gradient, the term involving the friciton force, and the tensor $W_{j k}$ which measures how rapidly the flow velocity varies in space and gives rise to plasma viscosity.

The ion analysis is slightly simpler since the ionelectron collision operator can be regarded as small, which implies that the friction is negligible in the ion kinetic problem. The analogue of (10) for ions is thus

$$
\begin{gathered}
C_{i i}\left(f_{i 1}\right)-\frac{e}{m_{i}} \mathbf{v} \times \mathbf{B} \cdot \frac{\partial f_{i 1}}{\partial \mathbf{v}} \\
=\left[\left(x^{2}-\frac{5}{2}\right) \mathbf{v} \cdot \nabla \ln T_{i}+\frac{m_{i}}{2 T_{i}}\left(v_{j} v_{k}-\frac{v_{i}^{2}}{3} \delta_{j k}\right) W_{j k}^{i}\right] f_{i 0},
\end{gathered}
$$

The equations (10) and (12) are linear, integrodifferential equations involving the complicated (but linearised) Coulomb collision operator. They are traditionally solved by expanding the distribution function in a suitable set of orthogonal functions, which converts the problem to an infinite set of coupled linear algebraic equations. A sufficiently accurate solution can be found by truncating this system of equations after only a few terms.

\section{III.B. Results}

Following Braginskii, we display the results in the limit $\rho / \lambda=1 / \Omega_{e} \tau_{e} \rightarrow 0$, which is the most interesting case for most situations in fusion and astrophysics. Here $\Omega_{e}=-e B / m_{e}$ is the electron gyrofrequency and

$$
\tau_{e}=\frac{12 \pi^{3 / 2}}{2^{1 / 2}} \frac{m_{e}^{1 / 2} T_{e}^{3 / 2} \epsilon_{0}^{2}}{n_{i} Z^{2} e^{4} \ln \Lambda}
$$

the electron collision time, with $Z=1$ the ion charge and $\ln \Lambda$ the Coulomb logarithm.

The force $\mathbf{F}_{e}$ acting on the electrons consists of a drag force and a thermal force

$$
\mathbf{F}_{e}=\mathbf{F}_{u}+\mathbf{F}_{T}
$$

$$
\begin{gathered}
\mathbf{F}_{u}=-\frac{m_{e} n_{e}}{\tau_{e}}\left(0.51 \mathbf{u}_{\|}+\mathbf{u}_{\perp}\right) \\
\mathbf{F}_{T}=-0.71 n_{e} \nabla_{\|} T_{e}+\frac{3 n_{e}}{2 \Omega_{e} \tau_{e}} \mathbf{b} \times \nabla T_{e}
\end{gathered}
$$

where $\mathbf{u}=\mathbf{V}_{e}-\mathbf{V}_{i}$ and $\mathbf{b}=\mathbf{B} / B$. Because of momentum conservation the force acting on the ions is equal and opposite, $\mathbf{F}_{i}=-\mathbf{F}_{e}$. The parallel friction coefficient in Eq (14) is seen to be smaller than the perpendicular one by a factor of 0.51 , which has to do with the fact that the collision frequency decreases with velocity, $\left(\tau_{e} \sim v^{3}\right)$, causing electrons with large parallel velocities to be more distorted from the Maxwellian distribution than slower ones. The fast electrons then contribute more to the relative velocity, and less to the friction. The parallel thermal force (15) is also a consequence of the circumstance that the collision frequency falls off with increasing energy, for consider a situation where the electron fluid is at rest with respect to the ion fluid, so that the drag force $\mathbf{F}_{u}$ vanishes. A typical ion is bombarded by electrons streaming along the field with their respective peculiar velocities. If there is a temperature gradient along the field, those electrons that travel in the direction of the temperature gradient come from a slightly colder region and will be more collisional than those going in the opposite direction. They will therefore exert a larger friction force on the ions, on which a thermal force therefore acts in the direction of $\nabla_{\|} T_{e}$. The corresponding force on the electrons (15) is in the opposite dirction. The second term is smaller than the first one by a factor of $1 / \Omega_{e} \tau_{e} \ll 1$.

The electron heat flux also has two pieces

$$
\begin{gathered}
\mathbf{q}_{e}=\mathbf{q}_{u}^{e}+\mathbf{q}_{T}^{e}, \\
\mathbf{q}_{u}^{e}=0.71 n_{e} T_{e} \mathbf{u}_{\|}-\frac{3 n_{e} T_{e}}{2 \Omega_{e} \tau_{e}} \mathbf{b} \times \mathbf{u}_{\perp}, \\
\mathbf{q}_{T}^{e}=-\kappa_{\|}^{e} \nabla_{\|} T_{e}-\kappa_{\wedge}^{e} \mathbf{b} \times \nabla T_{e}-\kappa_{\perp}^{e} \nabla_{\perp} T_{e},
\end{gathered}
$$

where the heat conductivities are

$$
\begin{gathered}
\kappa_{\|}^{e}=3.16 \frac{n_{e} T_{e} \tau_{e}}{m_{e}}, \\
\kappa_{\wedge}^{e}=-\frac{5 n_{e} T_{e}}{2 m_{e} \Omega_{e}}, \\
\kappa_{\perp}^{e}=4.66 \frac{n_{e} T_{e}}{m_{e} \Omega_{e}^{2} \tau_{e}},
\end{gathered}
$$

Physically, the first term in Eq. (17) has to do with the distortion of the distribution of fast electrons from a Maxwellian. In a coordinate system where $\mathbf{V}_{e}=0$, more fast electrons travel in the direction of $\mathbf{u}$ and more slow electrons in the direction of $-\mathbf{u}$, which gives rise to a heat flux. 
This effect does not arise for ions since the ionelectron collision frequency is independent of ion energy. The ion heat flux therefore only has terms related to $\nabla T_{i}$,

$$
\mathbf{q}_{i}=-\kappa_{\|}^{i} \nabla_{\|} T_{i}+\kappa_{\wedge}^{i} \mathbf{b} \times \nabla T_{i}-\kappa_{\perp}^{i} \nabla_{\perp} T_{i}
$$

where

$$
\begin{gathered}
\kappa_{\|}^{i}=3.9 \frac{n_{i} T_{i} \tau_{i}}{m_{i}} \\
\kappa_{\wedge}^{i}=\frac{5 n_{i} T_{i}}{2 m_{i} \Omega_{i}}, \\
\kappa_{\perp}^{i}=2 \frac{n_{i} T_{i}}{m_{i} \Omega_{i}^{2} \tau_{i}}
\end{gathered}
$$

with $\Omega_{i}=Z e B / m_{i}$ and

$$
\tau_{i i}=\frac{12 \pi^{3 / 2} m_{i}^{1 / 2} T_{i}^{3 / 2} \epsilon_{0}^{2}}{n_{i} Z^{2} e^{4} \ln \Lambda} .
$$

Note that the conductivities $\kappa_{\|}, \kappa_{\wedge}$ and $\kappa_{\perp}$ are consecutively separated by the large factor $\Omega \tau$ for both species. The first and third terms in the expressions for $\mathbf{q}_{T}^{e}$ and $\mathbf{q}_{i}$ are parallel to the gradients that drive them, and will therefore tend to relax these gradients. This relaxation occurs on very disparate time scales in the two directions. Perpendicular to the magnetic field, the ion contribution is dominant, since for both species the cross-field diffusion scales according as $\kappa_{\perp} \sim n \rho_{i}^{2} / \tau_{i}$, indicating a random walk with the step size $\rho_{i}$, which is much larger for the ions than for the electrons. Parallel to the field, on the other hand, the electron heat flux dominates, and scales as $\kappa_{\|} \sim n \lambda^{2} / \tau_{e}$ with a randomwalk step size equal to the mean free path. The latter is roughly the same for electrons and ions, but the collision time is much shorter for the electrons. Of intermediate magnitude is the diamagnetic heat flux term $\mathbf{q}_{\wedge}= \pm \kappa_{\wedge} \mathbf{b} \times \nabla T$, carrying heat across the field perpendicular to the gradient. This flux is not affected by collisions in the limit $\Omega_{i} \tau_{i} \gg 1$.

The heat exchanged between the species,

$$
Q_{i}=\int \frac{m_{i} v_{i}^{\prime 2}}{2} C_{i e}\left(f_{i}\right) d^{3} v=\frac{3 n_{e} m_{e}}{m_{i} \tau_{e}}\left(T_{e}-T_{i}\right),
$$

is a result of temperature equilibration on the slow time scale $m_{i} \tau_{e} / m_{e}$ and frictional generation of heat. The viscosity tensor is complicated and will not be treated here. Its form depends on whether the large- or smallflow ordering is adopted.

\section{III.C. Applications}

As already remarked, classical transport is relatively small across the magnetic field and is therefore usually of little concern. In most situations it is easily overwhelmed by neoclassical or turbulent transport. Along the field, however, the classical transport is very rapid and usually dominates over turbulence. The most important prediction of the classical theory is probably the electric (so-called Spitzer) conductivity, $\sigma$ regulating the current

$$
J_{\|}=-n_{e} e u_{\|}=\sigma E_{\|},
$$

that arises in response to an applied parallel electric field [5]. It is obtained from (14) as

$$
\sigma=1.96 \frac{n_{e} e^{2} \tau_{e}}{m_{e}}
$$

and is thus proportional to $T_{e}^{3 / 2}$ but independent of the density $n_{e}$.

The parallel electron heat conductivity (19) is very important in the tokamak edge, where it governs the heat flux to the divertor plates. This condutivity is proportional to $T_{e}^{5 / 2}$ but is independent of density. In situations where the heat flux is constant, it predicts a temperature profile $T_{e}(s) \sim s^{2 / 7}$ as a function of the arc length $s$ along the magnetic field. Parallel electron heat conduction is also important in space physics and astrophyiscs. It governs, for instance, the heat flux along magnetic loops in the solar corona.

The thermal force, which was discovered by Enskog (then a student) in 1911, has been used for isotope separation and plays an important role in plasmas with multiple ion species. The point is that the kinetic equation for hydrogenic ions in a plasma that also contains much heavier impurity ions is identical to the equation for electrons in a pure plasma (with the lighter ions playing the role of the electrons in a pure plasma). Many of Braginskii's results therefore carry over to the situation of an impure plasma and, in particular, there is a thermal force on heavy impurity ions in the direction of $\nabla_{\|} T_{i}$. This force pulls impurity ions out of the divertor into the main scrape-off-layer in a tokamak. In the transition region between the solar photosphere and the corona, there is a very strong vertical temperature gradient pulling out heavy ions and making the chemical composition of the solar wind different from that of the Sun.

\section{NEOCLASSICAL TRANSPORT}

Classical transport applies in all magnetic-field geometries as long as the mean free path is short. When it is, the transport is determined locally and does therefore not depend much on the macroscopic field structure. When the mean free path is long, however, the transport can become much stronger, which is the subject of neoclassical theory.

IV.A. Collisionality 
The physics of neoclassical transport in a tokamak depends decisively on the relative magnitude of the collision frequency $\nu$ and the transit frequency $\omega_{t}=$ $v_{T} / q R$, the so-called collisionality. Here $q=r B_{\varphi} / R B_{\theta}$ is the safety factor and $R$ the major radius of the tokamak. We note that the mean free path $\lambda=v_{T} / \nu$ is similar for electrons and ions since $\nu_{e e} / \nu_{i i} \sim v_{T e} / v_{T i}$, but may differ among ion species with very disparate masses. Highly charged impurities are more collisional than bulk ions and electrons. If the collisionality is large,

$$
\frac{L}{\lambda} \sim \frac{\nu}{v_{T} / q R} \gg 1
$$

the mean free path is shorter than the parallel distance around a flux surface $L \sim q R$, and the Braginskii fluid equations may be applied for the analysis. The particle orbits are then not fully completed by a typical thermal particle since its motion is disturbed by collisions before an orbit has been completed. This high-collisionality regime is called the Pfirsch-Schlüter regime.

In the opposite limit,

$$
\frac{\nu}{v_{T} / q R} \ll 1
$$

referred to as the banana-plateau regime, orbits are completed and short-mean-free-path closure of the fluid equations is inapplicable. The core of a tokamak is usually in this regime.

If the inverse aspect ratio is small, $\epsilon \ll 1$, the banana-plateau regime is subdivided into two regimes: the plateau regime

$$
\epsilon^{3 / 2} \ll \frac{\nu}{v_{T} / q R} \ll 1,
$$

and the banana regime

$$
\frac{\nu}{v_{T} / q R} \ll \epsilon^{3 / 2} .
$$

In the former, most circulating particle orbits are completed but trapped orbits are interrupted by collisions since the effective collision frequency, $\nu_{\text {eff }}=\nu / \epsilon$, required to scatter a trapped particle out of its magnetic well, $\Delta B / B \sim \epsilon$, is larger than the bounce frequency $\omega_{b} \sim \sqrt{\epsilon} v_{T} / q R$, i.e.,

$$
\nu_{*} \equiv \frac{\nu / \epsilon}{\omega_{b}}=\frac{\nu / \epsilon^{3 / 2}}{v_{T} / q R} \gg 1
$$

It is important to note that the effective collision frequency for scattering the velocity vector by an angle $\Delta \vartheta$ is $\nu /(\Delta \vartheta)^{2}$, since the pitch-angle scattering operator (8) is of second order. Trapped particles occupy the region $v_{\|} / v \sim \sqrt{\epsilon}$ in velocity space, so it is appropriate to take $\Delta \vartheta=\sqrt{\epsilon}$. The effective trapped-particle scattering frequency is thus substantially greater than $\nu$ if $\epsilon \ll 1$. Finally, in the banana regime, $\nu_{*} \ll 1$, the particle dynamics is virtually collisionless, and both types of orbits (trapped and circulating) can be completed.

The diffusivity of particles and heat is proportional to the collision frequency in the banana and PfirschSchlüter regimes (with different proportionality constants) but is independent of collisionality (forms a "plateau") in the plateau regime, see Fig 1. While this is strictly true in the limit $\epsilon \rightarrow 0$, at realistic aspect ratios the distinction between the regimes is blurred, and the plateau is difficult to discern.

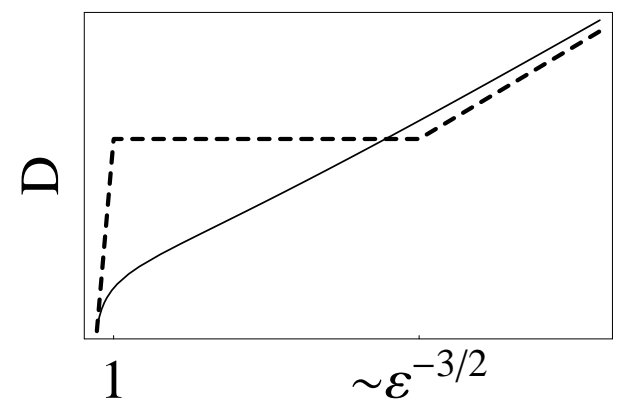

$\mathcal{V}_{*}$

Figure 1: Dependence of neoclassical diffusion coefficient on collisionality at large aspect ratio. The dashed curve represents the asymptotic limit $\epsilon \rightarrow 0$, while the solid curve represents $\epsilon=0.2$.

\section{IV.B. Flows within the flux surface}

The axisymmetric magnetic field of a tokamak has the form

$$
\mathbf{B}=I(\psi) \nabla \varphi+\nabla \varphi \times \nabla \psi
$$

where $\varphi$ is the toroidal angle measured in the direction of the plasma current and $\psi$ the poloidal flux function. Since the pressure

$$
p=\sum_{a} p_{a}=\sum_{a} n_{a} T_{a}
$$

is a flux function when the rotation velocity is subsonic so that $\mathbf{J} \times \mathbf{B}=\nabla p$, the diamagnetic current beomes

$$
\mathbf{J}_{\perp}=\frac{\mathbf{B} \times \nabla p}{B^{2}}=\left(\frac{I \mathbf{B}}{B^{2}}-R \hat{\varphi}\right) \frac{d p}{d \psi},
$$

and is generally not divergence-free, $\nabla \cdot \mathbf{J}_{\perp} \neq 0$, necessitating a parallel, so-called Pfirsch-Schlüter, current. Since

$$
0=\nabla \cdot\left(\frac{J_{\|} \mathbf{B}}{B}+\mathbf{J}_{\perp}\right)=\mathbf{B} \cdot \nabla\left(\frac{J_{\|}}{B}+\frac{I}{B^{2}} \frac{d p}{d \psi}\right)
$$


it follows that the quantity within the parentheses must be constant of flux surfaces, and the parallel current becomes

$$
J_{\|}=-\frac{I}{B} \frac{d p}{d \psi}+K(\psi) B,
$$

where $K(\psi)$ is an arbitrary integration constant. This is the "return current" necessary to close the diamagnetic current. A similar argument can be made for the flux $n_{a} \mathbf{V}_{a}$ for each species: its perpendicular component is given by the diamagnetic flux but is not divergence free, necessitating a parallel flux. The sum is equal to

$$
n_{a} \mathbf{V}_{a}=\omega_{a}(\psi) n_{a}(\psi) R \hat{\varphi}+K_{a}(\psi) \mathbf{B}
$$

with

$$
\omega_{a}(\psi)=-\frac{d \Phi}{d \psi}-\frac{1}{n_{a} e_{a}} \frac{d p_{a}}{d \psi},
$$

where $\Phi$ denotes the electrostatic potential. These relations hold in all collsionality regimes.

\section{PFIRSCH-SCHLÜTER TRANSPORT}

In the Pfirsch-Schlüter regime, the transport can mostly be calculated using Braginskii's equations. Although these do not depend on the geometry of the magnetic field, there is nevertheless an enhancement of the transport level in the tokamak as compared with a plasma in a straight magnetic field. On the fluid level, it has to do with the fact that a parallel Pfirsch-Schlüter flow must arise, as we have just seen. In the particle picture, the transport enhancement can be understood in terms of a simple random-walk argument.

Since the collision frequency exceeds the transit frequency, parallel particle motion is diffusive, with a diffusion coefficient

$$
D_{\|} \sim \lambda^{2} / \tau \sim v_{T}^{2} / \nu
$$

where $\lambda=v_{T} / \nu$ is the mean-free path. Thus, the time it takes for a particle to move around a flux surface is of the order

$$
\Delta t \sim \frac{(q R)^{2}}{D_{\|}} \sim \nu\left(\frac{q R}{v_{T}}\right)^{2},
$$

since the parallel distance is of the order $q R$. The crossfield transport is caused by the guiding-centre drift,

$$
\mathbf{v}_{d} \simeq-\frac{v^{2}+v_{\|}^{2}}{2 \Omega R} \hat{\mathbf{z}}
$$

which is vertical and therefore directed radially outward (say) if the particle is above the midplane and inward if it is below the midplane. As the particle diffuses in the parallel direction, the cross-field drift is sometimes outward and sometimes inward. This leads to a random walk in the radial direction, with a step length

$$
\Delta r \sim v_{d} \Delta t \sim \frac{\rho v_{T} \Delta t}{R},
$$

and a step time $\Delta t$. The resulting diffusion coefficient is thus

$$
D_{\perp} \sim \frac{(\Delta r)^{2}}{\Delta t} \sim \nu q^{2} \rho^{2},
$$

which is larger than the classical diffusion coefficient by a factor $q^{2}$.

To derive the transport mathematically using the results from Section III.B, we consider the ion heat flux. When the plasma flow velocity is ordered small, $E / B \sim \delta v_{T i}$, the ion energy equation (4) reduces to $\nabla \cdot \mathbf{q}_{i}=0$. Recalling (22), we see that if we expand the temperature,

$$
T_{i}=T_{i 0}+T_{i 1}+\ldots,
$$

in powers of $1 / \Omega_{i} \tau_{i}$, then in lowest order, $\nabla_{\|}\left(\kappa_{\|}^{i} \nabla_{\|} T_{i 0}\right)=0$, so

$$
T_{i 0}=T_{i 0}(\psi)
$$

Thus, on the fastest time scale parallel heat conduction makes the temperature uniform on flux surfaces. In next order, we have

$$
\nabla \cdot\left(\kappa_{\|}^{i} \nabla_{\|} T_{i 1}-\kappa_{\wedge}^{i} \mathbf{b} \times \nabla T_{i 0}\right)=0,
$$

so that

$$
\nabla_{\|} T_{i 1}=\frac{I \kappa_{\wedge}^{i}}{\kappa_{\|}^{i}} \frac{d T_{i 0}}{d \psi}+L_{i}(\psi) B,
$$

where the integration constant $L_{i}(\psi)$ can be determined from the relation $\left\langle B \nabla_{\|} T_{i 1}\right\rangle=0$. Here the angular brackets denote the so-called flux-surface average,

$$
\langle Q\rangle(\psi)=\oint \frac{Q(\psi, \theta)}{\mathbf{B} \cdot \nabla \theta} d \theta / \oint \frac{d \theta}{\mathbf{B} \cdot \nabla \theta}
$$

i.e., a volume average of the quantity $Q$ between two neighbouring flux surfaces. Since $\kappa_{\wedge}^{i} B$ is constant over the flux surface, it follows that

$$
\nabla_{\|} T_{i 1}=\frac{I \kappa_{\wedge}^{i}}{\kappa_{\|}^{i}}\left(1-\frac{B^{2}}{\left\langle B^{2}\right\rangle}\right) \frac{d T_{i 0}}{d \psi} .
$$

We see that $T_{i 1}$ varies over the flux surface. The mechanism is the same as that giving rise to the PfirschSchlüter current. The diamagnetic heat flux $\kappa_{\wedge}^{i} \mathbf{b} \times \nabla T_{i 0}$ is not divergence free, and must therefore be balanced by a parallel return flow, which, in turn, implies a small but important parallel temperature gradient. Since the latter (33) is positive on the inside of the torus and negative on the inside (assuming that $d T_{i 0} / d \psi<0$ ), the 
temperature is up-down asymmetric. It is now straightforward to construct the heat flux across the flux surface from (22) and (33),

$$
\begin{aligned}
& \mathbf{q}_{i} \cdot \nabla \psi=\left(-\kappa_{\perp}^{i} \nabla_{\perp} T_{i 0}+\kappa_{\wedge}^{i} \mathbf{b} \times \nabla T_{i 1}\right) \cdot \nabla \psi \\
= & -\left[\kappa_{\perp}^{i}|\nabla \psi|^{2}+\frac{\left(I \kappa_{\wedge}^{i}\right)^{2}}{\kappa_{\|}^{i}}\left(1-\frac{B^{2}}{\left\langle B^{2}\right\rangle}\right)\right] \frac{d T_{i 0}}{d \psi} .
\end{aligned}
$$

The first term is the classical cross-field heat flux, and the second term is the neoclassical Pfirsch-Schlüter heat flux, which arises entirely because of toroidicity. Both heat fluxes are ultimately driven by the radial temperature gradient, but the neoclassical heat flux is also fundamentally associated with a parallel gradient. In a large-aspect-ratio tokamak with circular cross section, the total flux-surface averaged heat flux becomes

$$
q_{i r}=\frac{\left\langle\mathbf{q}_{i} \cdot \nabla \psi\right\rangle}{|\nabla \psi|}=-\kappa_{\perp}^{i}\left(1+1.6 q^{2}\right) \frac{d T_{i 0}}{d r},
$$

where the first term is the classical and the second term the Pfirsch-Schlüter contribution.

\section{TRANSPORT IN THE BANANA REGIME}

When the mean free path is longer, the transport must be calculated kinetically, but since the gyroradius is supposed to be small, the full kinetic equation (1) can be reduced (by gyro-averaging) to the drift kinetic equation,

$$
\frac{\partial f_{a}}{\partial t}+\left(v_{\|} \mathbf{b}+\mathbf{v}_{d a}\right) \cdot \nabla f_{a}=C_{a}\left(f_{a}\right),
$$

where $\mathbf{v}_{d}$ is the drift velocity and the derivatives are taken at constant energy $H=m_{a} v^{2} / 2+e_{a} \Phi$ and magnetic moment $\mu=m_{a} v_{\perp}^{2} / 2 B$, so that the mirror force is contained in the parallel steaming term $v_{\|} \nabla_{\|} f_{a}$. In this equation, not all terms are equally large. As mentioned in the introduction, the first term on the left is ordered to be a factor $\delta^{2}$ smaller than the second one, and the magnetic drift term is by definition a factor $\delta$ smaller than parallel streaming, $v_{d} / v \sim \delta$. We may thus expand $f_{a}=f_{a 0}+f_{a 1}+\cdots$ and obtain

$$
\begin{gathered}
v_{\|} \nabla_{\|} f_{a 0}=C_{a}\left(f_{a 0}\right), \\
v_{\|} \nabla_{\|} f_{a 1}+\mathbf{v}_{d a} \cdot \nabla f_{a 0}=C_{a}\left(f_{a 1}\right),
\end{gathered}
$$

in zeroth and first order, respectively. It can relatively easily be shown that the only solutions of the first equations are Maxwellians that are constant on flux surfaces. This conclusion is reached by multiplying the equation by $\ln f_{a 0}$, integrating over velocity space, taking the flux-surface average, and using Boltzmann's Htheorem. It also follows from this argument that the electrostatic potential is approximately a flux function,

$$
\Phi=\Phi_{0}(\psi)+\Phi_{1}(\psi, \theta),
$$

with $\Phi_{1} / \Phi_{0} \sim \delta$

If an inductive electric field, $E_{\|}(A)=-\partial A_{\|} / \partial t$ is added to Eq (35), our remaining task is thus to solve the first-order equation

$v_{\|} \nabla_{\|} f_{a 1}-C_{a}\left(f_{a 1}\right)=-\left(\mathbf{v}_{d} \cdot \nabla \psi\right) \frac{\partial f_{a 0}}{\partial \psi}+\frac{e_{a} v_{\|} E_{\|}^{(A)}}{T_{a}} f_{a 0}$.

Since this equation is linear in $f_{a 1}$ and the driving terms on the right appear additively, we conclude that the radial density and temperature gradients in $f_{a 0}$ and the inductive electric field contribute separate and independent terms to $f_{a 1}$. In this equation no assumption has yet been made about collisionality, i.e., on the relative magnitude of the terms on the left. If the equation is solved under the assmption that the collision term domintates, then Pfirsch-Schlüter transport is recovered. If the first term instead dominates, banana transport ensues. Mathematically, one makes a subsidiary expansion of $f_{a 1}$ in the smallness of the collision frequency,

$$
f_{a 1}=f_{a 1}^{(0)}+f_{a 1}^{(1)}+\ldots
$$

which enables the equation to be solved analytically if the collision operator is simplified. The mathematics is somehwat complicated and will not be repeated here. The student who is interested in all the steps is encouraged to consult Ref [6].

\section{VI.A. Radial transport}

From the distribution funciton $f_{a 1}$ thus obtained, the radial particle and heat heat flux can be calculated. The results have been obtained in general axisymmetric geometry but are particularly simple in the case of a large-aspect-ratio tokamak with circular cross section. The largest flux is that of ion heat,

$$
q_{i r}=-n_{i} \chi_{i} \frac{d T_{i}}{d r}
$$

whose diffusivity is

$$
\chi_{i}=\frac{1.35 q^{2}}{\epsilon^{3 / 2}} \frac{T_{i}}{m_{i} \Omega_{i}^{2} \tau_{i}},
$$

and thus scales as the classical diffusivity $\rho_{i}^{2} / \tau_{i}$ enhanced by the factor $1.35 q^{2} / \epsilon^{3 / 2}$, which is usually in the range 10-100. The reason for this enhancement has to do with banana orbits. As already mentioned, particles with velocity vectors nearly perpendicular to the magnetic field, $\left|v_{\|}\right| / v_{\perp} \leq \epsilon^{1 / 2}$, are trapped in the relatively weak magnetic field on the outside of the torus and trace out banana orbits with a width

$$
\delta r_{b} \sim q \rho / \sqrt{\epsilon}
$$

cf Fig 2. The banana width $\delta r_{b}$ is thus considerably 


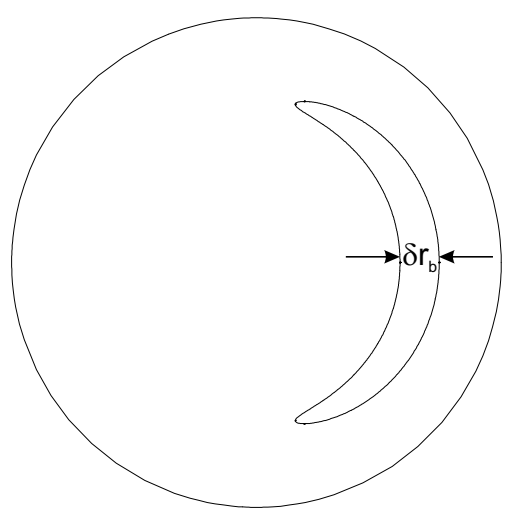

Figure 2: Banana orbit in a tokamak.

larger than the gyroradius, indicating a large randomwalk step size for these particles. The diffusivity (36) is of order

$$
\chi_{i} \sim f_{t}(\Delta x)^{2} \nu_{\text {eff }} .
$$

where the trapped fraction of the particles $f_{t} \sim \epsilon^{1 / 2}$ is small, but the step size $\Delta x \sim \delta r_{b}$ is large, and so is the effective collision frequency $\nu_{\text {eff }} \sim \nu / \epsilon$ of events causing a trapped ion to take a step of order $\delta r_{b}$.

The electron heat flux is also enhanced by a similar factor over the classical result, but is insignificant as it is smaller than the corresponding ion flux by a factor $\left(m_{e} / m_{i}\right)^{1 / 2}$. The ion and electron particle fluxes are equal (ambipolarity) and are both on the level of the electron heat flux. The only neoclassical cross-field flux that is experimentally relevant is thus the ion heat flux. Even this flux is usually overwhelmed by the turbulent transport, but can be of importance in spherical tokamaks and in transport barriers. The H-mode pedestal seems to exhibit neoclassical ion energy confinement.

\section{VI.B. Toroidal current}

The parallel current is very well described by neoclassical theory, which predicts two important effects: a reduction in the conductivity due to trapped particles and the existence of the so-called boostrap current. Both are of great experimental significance. When the drift kinetic equation is solved for $f_{i 1}$ and $f_{e 1}$, and the parallel current is calculated, one finds

$$
\begin{gathered}
J_{\|}=-\frac{f_{t} q n_{e} T_{e}}{\epsilon B}\left[1.66\left(1+\frac{T_{i}}{T_{e}}\right) \frac{d \ln n_{e}}{d r}\right. \\
\left.+0.47 \frac{d \ln T_{e}}{d r}-\frac{0.29}{T_{e}} \frac{d T_{i}}{d r}\right]+\left(1-1.31 f_{t}\right) \sigma E_{\|}^{(A)},
\end{gathered}
$$

for a large-aspect-ratio tokamak with circular cross section. The quantity $f_{t} \simeq 1.46 \epsilon^{1 / 2}$ denotes the "effective" fraction of trapped particles and appears in two places. It multiplies the entire first term, which constitutes the bootstrap current - a toroidal current that arises thanks to density and temperature gradients in the plasma. It also appears in the second term as a reduction of the Spitzer conductivity $\sigma$ caused by the fact that trapped particles cannot contribute to the parallel current.

Physically, the bootstrap current has its root in a diamagnetic effect of the banana orbits. Because of their width $\delta r_{b} \sim q \rho / \epsilon^{1 / 2}$, the co-current-moving, trapped population on a given flux surface is larger than the counter-moving one by an amount

$$
-\epsilon^{1 / 2} \frac{d n}{d r} \delta r_{b}
$$

where $\epsilon^{1 / 2}$ is the approximate fraction of trapped particles. These are in collisional equilibrium with the passing ones, whose co-passing population therefore exceeds the counter-passing one by

$$
-\frac{d n}{d r} \delta r_{b}
$$

The resulting current is of the order

$$
J_{B S} \sim-v_{T} e_{a} \frac{d n}{d r} \delta r_{b} \sim-\frac{q T_{a}}{\epsilon^{1 / 2} B} \frac{d n_{a}}{d r} .
$$

Thus, at large aspect ratio the bootstrap current is mostly carried by the passing particles, although it is ultimately caused by the diamagnetic effect of the trapped ones.

The bootstrap current is thus of order

$$
J_{B S} \sim \frac{\epsilon^{1 / 2} p}{r B_{p}},
$$

where $B_{p}=\epsilon B / q$ is the poloidal field, and compares in the following way with the Ohmic current,

$$
J_{B S} / J_{O H} \sim \epsilon^{1 / 2} \beta_{p},
$$

where $\beta_{p}=2 \mu_{0} p / B_{p}^{2}$ is the poloidal beta. In the standard tokamak ordering $\beta \sim \epsilon^{2} \Rightarrow \beta_{p} \sim 1$, the bootstrap current is thus formally smaller than the Ohmic current by a factor $\epsilon^{1 / 2}$. In practice, $\beta$ and $\epsilon^{1 / 2}$ are sometimes not very small, and the bootstrap current is often comparable to, or even larger than, the Ohmic current. It is of great importance for the prospects of the tokamak to be an economic power source.

\section{VI.C. Plasma rotation}

We have already shown that the flow within the flux surface of each species is given by Eq (30), where the constant $K_{a}$ must be calculated from kinetic theory. For ions this constant determines the poloidal plasma rotation, which is equal to

$$
V_{i \theta}=\frac{1.17}{m_{i} \Omega_{i}} \frac{d T_{i}}{d r}
$$


for a circular, large-aspect-ratio tokamak in the banana regime. Contrary to widespread belief, the poloidal rotation is independent of the radial electric field. In fact, this field only plays a minor role in neoclassical theory for axisymmetric plasmas. In the quasi-steady state described by the transport ordering, it does not affect cross-field transport or poloidal rotation. The only observable quantity where it shows up is the toroidal rotation frequency (31), where it appears in the same way for all species. Of course, there is a good reason for this. Suppose that we make a transformation from the laboratory frame to a frame rotating at the velocity

$$
\mathbf{V}=-\hat{\boldsymbol{\varphi}} R \frac{d \Phi_{0}}{d \psi}
$$

of some flux surface $\psi$. The electric field measured in this frame vanishes on the flux surface in question since

$$
\mathbf{E}^{\prime}=\mathbf{E}+\mathbf{V} \times \mathbf{B} \simeq-\nabla \Phi_{0}+\mathbf{V} \times \mathbf{B}=0,
$$

and the equation of motion for each species acquires new terms corresponding to the centrifugal force and the Coriolis force. These terms are, however, small if V is smaller than the thermal speed, in which case the only consequence of the coordinate transformation is to eliminate the radial electric field. Thus, as long as the flow velocity is small, the radial electric field cannot affect neoclassical transport.

For this reason, the transport in a tokamak is intrinsically ambipolar, i.e., the radial electron and ion particle fluxes are always the same, regardless of the radial electric field, as long as the transport ordering is satisfied. The toroidal rotation frequency (31) can therefore attain any value: the plasma rotates freely in the toroidal direction. The situation is very different in non-axisymmetric systems such as stellarators, where ambipolarity is only attained at a certain value of the radial electric field. A stellarator plasma cannot rotate freely [7].

\section{DISCUSSION}

Not only the neoclassical theory, but most of the theory of magnetically confined plasmas relies on an expansion in $\delta \ll 1$, and one can thus distinguish between plasma physics phenomena according to the order in which they first appear in the expansion. In zeroth order one finds, among other things, that the distribution function of each species must be Maxwellian, that flux surfaces should be isothermal, that an axisymmetric plasma is free to rotate toroidally, that its equilbrium and stability properties are predicted by MHD, and that the parallel electric conductivity is given by the Spitzer value with a reduction due to particle trapping. In first order, the bootstrap current and neoclassical poloidal rotation appear, and in second order there is radial transport - either caused by collisions as in the neoclassical theory or by gyrokinetic turbulence. It is interesting to note that the robustness of the theoretical predictions decreases with increasing order in the expansion. The zeroth-order predictions are very robust in the sense that they are hardly affected by turbulence and are very well borne out in experiments. The first-order results are also broadly in line with observations, whilst the cross-field transport, which is of second order, is famously difficult to predict with confidence. The neoclassial cross-field transprort is usually overwhelmed by turbulent transport, but this is (in gyrokinetics) also of second order in the gyroradius and therefore sensitive to details in the mathematical treatment and the physical conditions prevailing in the plasma.

\section{FURTHER READING}

A more complete exposition can be found in the author's book [6] and in the review by Hinton and Hazeltine [8]. Classical transport is very well described in the reviews by Braginskii [3] and Hinton [9].

\section{REFERENCES}

1. S. CHAPMAN, Phil. Trans. R. Soc. London Ser. A 98, 1 (1916).

2. D. ENSKOG, Kinetische Theorie der Vorgänge in mässig verdünnten Gasen (Almqvist \& Wiksell, Uppsala, 1917), Inaugural dissertation, Uppsala University; Arkiv för matematik, astronomi och fysik 16, 16, 1 (1922).

3. S.I. BRAGINSKII, in Reviews of Plasma Physics, edited by M. A. Leontovich (Consultants Bureau, New York, 1965), Vol. 1, p. 205.

4. P.J. CATTO and A.N. SIMAKOV, Phys. Plasmas 11, 90 (2004).

5. L. SPITZER and R. HÄRM, Phys. Rev. 89, 977 (1953).

6. P. HELANDER and D.J. SIGMAR, Collisional transport in magnetized plasmas (Cambridge University Press, 2002).

7. P. HELANDER and A.N. SIMAKOV, Phys. Rev. Lett. 101, 145003 (2008).

8. F.L. HINTON and R.D. HAZELTINE, Rev. Mod. Phys 48, 239 (1976).

9. F.L. HINTON, in Handbook of Plasma Physics, edited by M.N. Rosenbluth and R.Z. Sagdeev, Vol. 1, p. 147 (North-Holland, Amsterdam, 1983). 